\title{
AUTOMORPHISMS OF FINITE ABELIAN GROUPS
}

\author{
CHRISTOPHER J. HILLAR AND DARREN L. RHEA
}

\section{INTRODUCTION}

In introductory abstract algebra classes, one typically encounters the classification of finite Abelian groups [2]:

Theorem 1.1. Let $G$ be a finite Abelian group. Then $G$ is isomorphic to a product of groups of the form

$$
H_{p}=\mathbb{Z} / p^{e_{1}} \mathbb{Z} \times \cdots \times \mathbb{Z} / p^{e_{n}} \mathbb{Z}
$$

in which $p$ is a prime number and $1 \leq e_{1} \leq \cdots \leq e_{n}$ are positive integers.

Much less known, however, is that there is a description of $\operatorname{Aut}(G)$, the automorphism group of $G$. The first compete characterization that we are aware of is contained in a paper by Ranum [1] near the turn of the last century. Beyond this, however, there are few other expositions [4]. Our goal is to fill this gap, thereby providing a much needed accessible and modern treatment.

Our characterization of $\operatorname{Aut}(G)$ is accomplished in three main steps. The first observation is that it is enough to work with the simpler groups $H_{p}$. This reduction is carried out by appealing to a fact about product automorphisms for groups with relatively prime numbers of elements (Lemma 2.1). Next, we use Theorem 3.3 to describe the endomorphism ring of $H_{p}$ as a quotient of a matrix subring of $\mathbb{Z}^{n \times n}$. And finally, the units $\operatorname{Aut}\left(H_{p}\right) \subset \operatorname{End}\left(H_{p}\right)$ are identified from this construction.

As a consequence of our investigation, we readily obtain an explicit formula for the number of elements of $\operatorname{Aut}(G)$ for any finite Abelian group $G$ (see also [3]).

\section{Product Automorphisms}

Let $G=H \times K$ be a product of groups $H$ and $K$, in which the orders of $H$ and $K$ are relatively prime positive integers. It is natural to ask how the automorphisms of $G$ are related to those of $H$ and $K$.

Lemma 2.1. Let $H$ and $K$ be finite groups with relatively prime orders. Then

$$
\operatorname{Aut}(H) \times \operatorname{Aut}(K) \cong \operatorname{Aut}(H \times K) .
$$

Proof. We exhibit a homomorphism $\phi: \operatorname{Aut}(H) \times \operatorname{Aut}(K) \rightarrow \operatorname{Aut}(H \times K)$ as follows. Let $\alpha \in \operatorname{Aut}(H)$ and $\beta \in \operatorname{Aut}(K)$. Then, as is easily seen, an automorphism $\phi(\alpha, \beta)$ of $H \times K$ is given by

$$
\phi(\alpha, \beta)(h, k)=(\alpha(h), \beta(k)) .
$$

Let $\operatorname{id}_{H} \in \operatorname{Aut}(H)$ and $\operatorname{id}_{K} \in \operatorname{Aut}(K)$ be the identity automorphisms of $H$ and $K$, respectively. To prove that $\phi$ is a homomorphism, notice that $\phi\left(\mathrm{id}_{H}, \mathrm{id}_{K}\right)=\mathrm{id}_{H \times K}$ and that

$$
\phi\left(\alpha_{1} \alpha_{2}, \beta_{1} \beta_{2}\right)(h, k)=\left(\alpha_{1} \alpha_{2}(h), \beta_{1} \beta_{2}(k)\right)=\phi\left(\alpha_{1}, \beta_{1}\right) \phi\left(\alpha_{2}, \beta_{2}\right)(h, k),
$$

for all $\alpha_{1}, \alpha_{2} \in \operatorname{Aut}(H), \beta_{1}, \beta_{2} \in \operatorname{Aut}(K)$, and $h \in H, k \in K$. 
We next verify that $\phi$ is an isomorphism. It is clear that $\phi$ is injective; thus we are left with showing surjectivity. Let $n=|H|, m=|K|$, and write $\pi_{H}$ and $\pi_{K}$ for the standard projection homomorphisms $\pi_{H}: H \times K \rightarrow H$ and $\pi_{K}: H \times K \rightarrow$ $K$. Fix $\omega \in \operatorname{Aut}(H \times K)$, and consider the homomorphism $\gamma: K \rightarrow H$ given by $\gamma(k)=\pi_{H}\left(w\left(1_{H}, k\right)\right)$, in which $1_{H}$ is the identity element of $H$. Notice that $\left\{k^{n}: k \in K\right\} \subseteq \operatorname{ker} \gamma$ since

$$
1_{H}=\pi_{H}\left(w\left(1_{H}, k\right)\right)^{n}=\pi_{H}\left(w\left(1_{H}, k\right)^{n}\right)=\pi_{H}\left(w\left(1_{H}, k^{n}\right)\right)=\gamma\left(k^{n}\right) .
$$

Also, since $m$ and $n$ are relatively prime, the set $\left\{k^{n}: k \in K\right\}$ consists of $m$ elements. Consequently, it follows that $\operatorname{ker} \gamma=K$ and $\gamma$ is the trivial homomorphism. Similarly, $\delta: H \rightarrow K$ given by $\delta(h)=\pi_{K}\left(w\left(h, 1_{K}\right)\right)$ is trivial.

Finally, define endomorphisms of $H$ and $K$ as follows:

$$
\omega_{H}(h)=\pi_{H}\left(\omega\left(h, 1_{K}\right)\right), \omega_{K}(k)=\pi_{K}\left(\omega\left(1_{H}, k\right)\right) .
$$

From this construction and the above arguments, we have

$$
\omega(h, k)=\omega\left(h, 1_{K}\right) \cdot \omega\left(1_{H}, k\right)=\left(\omega_{H}(h), \omega_{K}(k)\right)=\phi\left(\omega_{H}, \omega_{K}\right)(h, k)
$$

for all $h \in H$ and $k \in K$. It remains to prove that $\omega_{H} \in \operatorname{Aut}(H)$ and $\omega_{K} \in$ $\operatorname{Aut}(K)$, and for this it suffices that $\omega_{H}$ and $\omega_{K}$ are injective (since both $H$ and $K$ are finite). To this end, suppose that $\omega_{H}(h)=1_{H}$ for some $h \in H$. Then $w\left(h, 1_{K}\right)=\left(w_{H}(h), w_{K}\left(1_{K}\right)\right)=\left(1_{H}, 1_{K}\right)$, so $h=1_{H}$ by injectivity of $w$. A similar argument shows that $\omega_{K} \in \operatorname{Aut}(K)$, and this completes the proof.

Let $p$ be a prime number. The order of $H_{p}=\mathbb{Z} / p^{e_{1}} \mathbb{Z} \times \cdots \times \mathbb{Z} / p^{e_{n}} \mathbb{Z}$ is easily seen to be $p^{e_{1}+\cdots+e_{n}}$. As $G$ is isomorphic to a finite product of $H_{p}$ over a distinct set of primes $p$, Lemma 2.1 implies that $\operatorname{Aut}(G)$ is simply the product of $\operatorname{Aut}\left(H_{p}\right)$ over the same set of primes. We will, therefore, devote our attention to computing $\operatorname{Aut}\left(H_{p}\right)$ for primes $p$ and integers $1 \leq e_{1} \leq \cdots \leq e_{n}$.

\section{ENDOMORPHisms of $H_{p}$}

In order to carry out our characterization, it will be necessary to give a description of $E_{p}=\operatorname{End}\left(H_{p}\right)$, the endomorphism ring of $H_{p}$. Elements of $E_{p}$ are group homomorphisms from $H_{p}$ into itself, with ring multiplication given by composition and addition given naturally by $(A+B)(h):=A(h)+B(h)$ for $A, B \in \operatorname{End}\left(H_{p}\right)$ and $h \in H_{p}$. These rings behave much like matrix rings with some important differences that we discuss below.

The cyclic group $C_{p^{e_{i}}}=\mathbb{Z} / p^{e_{i}} \mathbb{Z}$ corresponds to the additive group for arithmetic modulo $p^{e_{i}}$, and we let $g_{i}$ denote the natural (additive) generator for $C_{p_{i} e_{i}}$. Specifically, these elements $g_{i}$ can be viewed as the classes

$$
\overline{1}=\left\{x \in \mathbb{Z}: x \equiv 1\left(\bmod p^{e_{i}}\right)\right\}
$$

of integers with remainder 1 upon division by $p^{e_{i}}$.

Under this representation, an element of $H_{p}$ is a column vector $\left(\bar{h}_{1}, \ldots, \bar{h}_{n}\right)^{T}$ in which each $\bar{h}_{i} \in \mathbb{Z} / p^{e_{i}} \mathbb{Z}$ and $h_{i} \in \mathbb{Z}$ is an integral representative. With these notions in place, we define the following set of matrices.

\section{Definition 3.1.}

$$
R_{p}=\left\{\left(a_{i j}\right) \in \mathbb{Z}^{n \times n}: p^{e_{i}-e_{j}} \mid a_{i j} \text { for all } i \text { and } j \text { satisfying } 1 \leq j \leq i \leq n\right\} .
$$


As a simple example, take $n=3$ with $e_{1}=1, e_{2}=2$, and $e_{3}=5$. Then

$$
R_{p}=\left\{\left[\begin{array}{ccc}
b_{11} & b_{12} & b_{13} \\
b_{21} p & b_{22} & b_{23} \\
b_{31} p^{4} & b_{32} p^{3} & b_{33}
\end{array}\right]: b_{i j} \in \mathbb{Z}\right\} .
$$

In general, it is clear that $R_{p}$ is closed under addition and contains the $n \times n$ identity matrix $I$. It turns out that matrix multiplication also makes this set into a ring as the following lemma demonstrates.

Lemma 3.2. $R_{p}$ forms a ring under matrix multiplication.

Proof. Let $A=\left(a_{i j}\right) \in R_{p}$. The condition that $p^{e_{i}-e_{j}} \mid a_{i j}$ for all $i \geq j$ is equivalent to the existence of a decomposition

$$
A=P A^{\prime} P^{-1},
$$

in which $A^{\prime} \in \mathbb{Z}^{n \times n}$ and $P=\operatorname{diag}\left(p^{e_{1}}, \ldots, p^{e_{n}}\right)$ is diagonal. In particular, if $A, B \in R_{p}$, then $A B=\left(P A^{\prime} P^{-1}\right)\left(P B^{\prime} P^{-1}\right)=P A^{\prime} B^{\prime} P^{-1} \in R_{p}$ as required.

Let $\pi_{i}: \mathbb{Z} \rightarrow \mathbb{Z} / p^{e_{i}} \mathbb{Z}$ be the standard quotient mapping $\pi_{i}(h)=\bar{h}$, and let $\pi: \mathbb{Z}^{n} \rightarrow H_{p}$ be the homomorphism given by

$$
\pi\left(h_{1}, \ldots, h_{n}\right)^{T}=\left(\pi_{1}\left(h_{1}\right), \ldots, \pi_{n}\left(h_{n}\right)\right)^{T}=\left(\bar{h}_{1}, \ldots, \bar{h}_{n}\right)^{T} .
$$

We may now give a description of $E_{p}$ as a quotient of the matrix ring $R_{p}$. In words, the result says that an endomorphism of $H_{p}$ is multiplication by a matrix $A \in R_{p}$ on a vector of integer representatives, followed by an application of $\pi$.

Theorem 3.3. The map $\psi: R_{p} \rightarrow \operatorname{End}\left(H_{p}\right)$ given by

$$
\psi(A)\left(\bar{h}_{1}, \ldots, \bar{h}_{n}\right)^{T}=\pi\left(A\left(h_{1}, \ldots, h_{n}\right)^{T}\right)
$$

is a surjective ring homomorphism.

Proof. Let us first verify that $\psi(A)$ is a well-defined map from $H_{p}$ to itself. Let $A=\left(a_{i j}\right) \in R_{p}$, and suppose that $\left(\bar{r}_{1}, \ldots, \bar{r}_{n}\right)^{T}=\left(\bar{s}_{1}, \ldots, \bar{s}_{n}\right)^{T}$ for integers $r_{i}, s_{i}$ (so that $p^{e_{i}} \mid r_{i}-s_{i}$ for all $\left.i\right)$. The $k$ th vector entry of the difference $\pi\left(A\left(r_{1}, \ldots, r_{n}\right)^{T}\right)-$ $\pi\left(A\left(s_{1}, \ldots, s_{n}\right)^{T}\right)$ is

$$
\begin{aligned}
\pi_{k}\left(\sum_{i=1}^{n} a_{k i} r_{i}\right)-\pi_{k}\left(\sum_{i=1}^{n} a_{k i} s_{i}\right) & =\pi_{k}\left(\sum_{i=1}^{n} a_{k i} r_{i}-\sum_{i=1}^{n} a_{k i} s_{i}\right) \\
& =\sum_{i=1}^{n} \pi_{k}\left(\frac{a_{k i}}{p^{e_{k}-e_{i}}} \cdot p^{e_{k}-e_{i}}\left(r_{i}-s_{i}\right)\right) \\
& =\overline{0}
\end{aligned}
$$

since $p^{e_{k}} \mid p^{e_{k}-e_{i}}\left(r_{i}-s_{i}\right)$ for $k \geq i$ and $p^{e_{k}} \mid\left(r_{i}-s_{i}\right)$ when $k<i$. Next, since $\pi$ and $A$ are both linear, it follows that $\psi(A)$ is linear. Thus, $\psi(A) \in \operatorname{End}\left(H_{p}\right)$ for all $A \in R_{p}$.

To prove surjectivity of the map $\psi$, let $w_{i}=\left(0, \ldots, g_{i}, \ldots, 0\right)^{T}$ be the vector with $g_{i}$ in the $i$ th component and zeroes everywhere else. An endomorphism $M \in$ $\operatorname{End}\left(H_{p}\right)$ is determined by where it sends each $w_{i}$; however, there isn't complete 
freedom in the mapping of these elements. Specifically, suppose that $M\left(w_{j}\right)=$ $\left(\bar{h}_{1 j}, \ldots, \bar{h}_{n j}\right)^{T}=\pi\left(h_{1 j}, \ldots, h_{n j}\right)^{T}$ for integers $h_{i j}$. Then,

$$
0=M(0)=M\left(p^{e_{j}} w_{j}\right)=\underbrace{M w_{j}+\cdots+M w_{j}}_{p^{e_{j}}}=\left(\overline{p^{e_{j}} h_{1 j}}, \ldots, \overline{p^{e_{j}} h_{n j}}\right)^{T} .
$$

Consequently, it follows that $p^{e_{i}} \mid p^{e_{j}} h_{i j}$ for all $i$ and $j$, and therefore $p^{e_{i}-e_{j}} \mid h_{i j}$ when $i \geq j$. Forming the matrix $H=\left(h_{i j}\right) \in R_{p}$, we have $\psi(H)=M$ by construction, and this proves that $\psi$ is surjective.

Finally, we need to show that $\psi$ is a ring homomorphism. Clearly, from the definition, $\psi(I)=\mathrm{id}_{E_{p}}$, and also $\psi(A+B)=\psi(A)+\psi(B)$. If $A, B \in R_{p}$, then a straightforward calculation reveals that $\psi(A B)$ is the endomorphism composition $\psi(A) \circ \psi(B)$ by the properties of matrix multiplication. This completes the proof.

Given this description of $\operatorname{End}\left(H_{p}\right)$, one can characterize those endomorphisms giving rise to elements in $\operatorname{Aut}\left(H_{p}\right)$. Before beginning this discussion, let us first calculate the kernel of the map $\psi$ defined in Theorem 3.3.

Lemma 3.4. The kernel of $\psi$ is given by the set of matrices $A=\left(a_{i j}\right) \in R_{p}$ such that $p^{e_{i}} \mid a_{i j}$ for all $i, j$.

Proof. As before, let $w_{j}=\left(0, \ldots, g_{j}, \ldots, 0\right)^{T} \in H_{p}$ be the vector with $g_{j}$ in the $j$ th component and zeroes everywhere else. If $A=\left(a_{i j}\right) \in R_{p}$ has the property that each $a_{i j}$ is divisible by $p^{e_{i}}$, then

$$
\psi(A) w_{j}=\left(\pi_{1}\left(a_{1 j}\right), \ldots, \pi_{n}\left(a_{n j}\right)\right)=0 .
$$

In particular, since each $h \in H_{p}$ is a $\mathbb{Z}$-linear combination of the $w_{j}$, it follows that $\psi(A) h=0$ for all $h \in H_{p}$. This proves that $A \in \operatorname{ker} \psi$.

Conversely, suppose that $A=\left(a_{i j}\right) \in \operatorname{ker} \psi$, so that $\psi(A) w_{j}=0$ for each $w_{j}$. Then, from the above calculation, each $a_{i j}$ is divisible by $p^{e_{i}}$. This proves the lemma.

Theorem 3.3 and Lemma 3.4 together give an explicit characterization of the ring $\operatorname{End}\left(H_{p}\right)$ as a quotient $R_{p} / \operatorname{ker} \psi$. Following this discussion, we now calculate the units $\operatorname{Aut}\left(H_{p}\right)$. The only additional tool that we require is the following fact from elementary matrix theory.

Lemma 3.5. Let $A \in \mathbb{Z}^{n \times n}$ with $\operatorname{det}(A) \neq 0$. Then there exists a unique matrix $B \in \mathbb{Q}^{n \times n}$ (called the adjugate of $A$ ) such that $A B=B A=\operatorname{det}(A) I$, and moreover $B$ has integer entries.

Writing $\mathbb{F}_{p}$ for the field $\mathbb{Z} / p \mathbb{Z}$, the following is a complete description of $\operatorname{Aut}\left(H_{p}\right)$.

Theorem 3.6. An endomorphism $M=\psi(A)$ is an automorphism if and only if $A(\bmod p) \in \mathrm{GL}_{n}\left(\mathbb{F}_{p}\right)$.

Proof. We begin with a short interlude. Fix a matrix $A \in R_{p}$ with $\operatorname{det}(A) \neq 0$. Lemma 3.5 tells us that there exists a matrix $B \in \mathbb{Z}^{n \times n}$ such that $A B=B A=$ $\operatorname{det}(A) I$. We would like to show that $B$ is actually an element of $R_{p}$. For the proof, express $A=P A^{\prime} P^{-1}$ for some $A^{\prime} \in \mathbb{Z}^{n \times n}$, and let $B^{\prime} \in \mathbb{Z}^{n \times n}$ be such that $A^{\prime} B^{\prime}=B^{\prime} A^{\prime}=\operatorname{det}\left(A^{\prime}\right) I$ (again using Lemma 3.5). Notice that $\operatorname{det}(A)=\operatorname{det}\left(A^{\prime}\right)$. Let $C=P B^{\prime} P^{-1}$ and observe that

$$
A C=P A^{\prime} B^{\prime} P^{-1}=\operatorname{det}(A) I=P B^{\prime} A^{\prime} P^{-1}=C A .
$$


By the uniqueness of $B$ from the lemma, it follows that $B=C=P B^{\prime} P^{-1}$, and thus $B$ is in $R_{p}$, as desired.

Returning to the proof of the theorem $(\Leftarrow)$, suppose that $p \nmid \operatorname{det}(A)$ (so that $A$ $\left.(\bmod p) \in \mathrm{GL}_{n}\left(\mathbb{F}_{p}\right)\right)$, and let $s \in \mathbb{Z}$ be such that $s$ is the inverse of $\operatorname{det}(A)$ modulo $p^{e_{n}}\left(\right.$ such an integer $s$ exists since $\left.\operatorname{gcd}\left(\operatorname{det}(A), p^{e_{n}}\right)=1\right)$. Notice that we also have $\operatorname{det}(A) \cdot s \equiv 1\left(\bmod p^{e_{j}}\right)$ whenever $1 \leq j \leq n$. Let $B$ be the adjugate of $A$ as in Lemma 3.5. We now define an element of $R_{p}$,

$$
A^{(-1)}:=s \cdot B,
$$

whose image under $\psi$ is the inverse of the endomorphism represented by $A$ :

$$
\psi\left(A^{(-1)} A\right)=\psi\left(A A^{(-1)}\right)=\psi(s \cdot \operatorname{det}(A) I)=\operatorname{id}_{E_{p}} .
$$

This proves that $\psi(A) \in \operatorname{Aut}\left(H_{p}\right)$.

Conversely, if $\psi(A)=M$ and $\psi(C)=M^{-1} \in \operatorname{End}\left(H_{p}\right)$ exists, then

$$
\psi(A C-I)=\psi(A C)-\mathrm{id}_{E_{p}}=0 .
$$

Hence, $A C-I \in \operatorname{ker} \psi$. From the kernel calculation in Lemma 3.4, it follows that $p \mid A C-I$ (entrywise), and so $A C \equiv I(\bmod p)$. Therefore,

$$
1 \equiv \operatorname{det}(A C) \equiv \operatorname{det}(A) \operatorname{det}(C) \quad(\bmod p) .
$$

In particular, $p \nmid \operatorname{det}(A)$, and the theorem follows.

As a simple application of the above discussion, consider the case when $e_{i}=1$ for $i=1, \ldots, n$. Here, $H_{p}$ can be viewed as the familiar vector space $\mathbb{F}_{p}^{n}$ and $\operatorname{End}\left(H_{p}\right)$ is isomorphic to the ring $\mathrm{M}_{n}\left(\mathbb{F}_{p}\right)$ of $n \times n$ matrices with coefficients in the field $\mathbb{F}_{p}$. Theorem 3.6 is then simply the statement that $\operatorname{Aut}\left(H_{p}\right)$ corresponds to the set of invertible matrices $\mathrm{GL}_{n}\left(\mathbb{F}_{p}\right)$.

\section{Counting the Automorphisms of $H_{p}$}

To further convince the reader of the usefulness of Theorem 3.6, we will briefly explain how to count the number of elements in $\operatorname{Aut}\left(H_{p}\right)$ using our characterization. Appealing to Lemma 2.1, one then finds an explicit formula for the number of automorphisms of any finite Abelian group. The calculation proceeds in two stages: (1) finding all elements of $\mathrm{GL}_{n}\left(\mathbb{F}_{p}\right)$ that can be extended to a matrix $A \in R_{p}$ that represents an endomorphism, and then (2) calculating all the distinct ways of extending such an element to an endomorphism.

Define the following $2 n$ numbers:

$$
d_{k}=\max \left\{l: e_{l}=e_{k}\right\}, c_{k}=\min \left\{l: e_{l}=e_{k}\right\} .
$$

Since $e_{k}=e_{k}$, we have $d_{k} \geq k$ and $c_{k} \leq k$. We need to find all $M \in \mathrm{GL}_{n}\left(\mathbb{F}_{p}\right)$ of the form

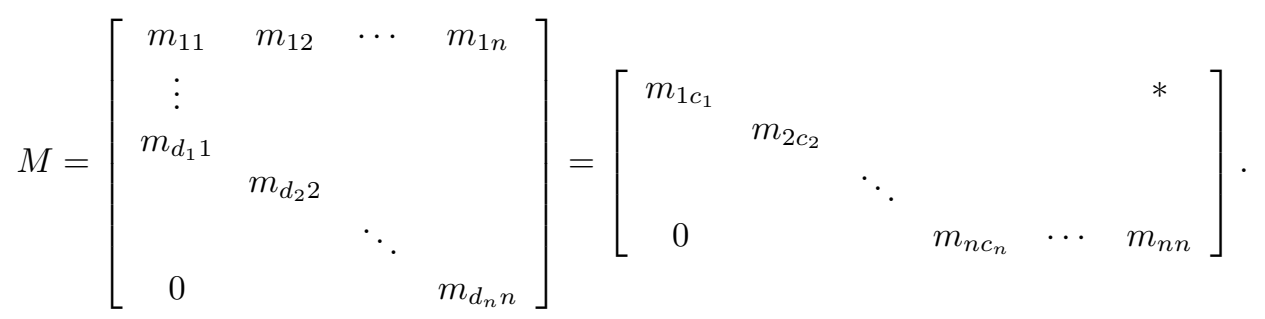


These number

$$
\prod_{k=1}^{n}\left(p^{d_{k}}-p^{k-1}\right)
$$

since we only need linearly independent columns. Next, to extend each element $m_{i j}$ from $\bar{m}_{i j} \in \mathbb{Z} / p \mathbb{Z}$ to $\bar{a}_{i j} \in p^{e_{i}-e_{j}} \mathbb{Z} / p^{e_{i}} \mathbb{Z}$ such that

$$
a_{i j} \equiv m_{i j}(\bmod p)
$$

there are $p^{e_{j}}$ ways to do this to the necessary zeroes (i.e., when $e_{i}>e_{j}$ ), since any element of $p^{e_{i}-e_{j}} \mathbb{Z} / p^{e_{i}} \mathbb{Z}$ will do. Additionally, there are $p^{e_{i}-1}$ ways at the not necessarily zero entries $\left(e_{i} \leq e_{j}\right)$, since we may add any element of $p \mathbb{Z} / p^{e_{i}} \mathbb{Z}$. This proves the following result.

Theorem 4.1. The Abelian group $H_{p}=\mathbb{Z} / p^{e_{1}} \mathbb{Z} \times \cdots \times \mathbb{Z} / p^{e_{n}} \mathbb{Z}$ has

$$
\left|\operatorname{Aut}\left(H_{p}\right)\right|=\prod_{k=1}^{n}\left(p^{d_{k}}-p^{k-1}\right) \prod_{j=1}^{n}\left(p^{e_{j}}\right)^{n-d_{j}} \prod_{i=1}^{n}\left(p^{e_{i}-1}\right)^{n-c_{i}+1} \text {. }
$$

\section{REFERENCES}

[1] A. Ranum. The group of classes of congruent matrices with application to the group of isomorphisms of any abelian group. Trans. Amer. Math. Soc. 8 (1907) 71-91.

[2] S. Lang, Algebra 3rd ed., Addison-Wesley Publishing Company, New York, 1993.

[3] J.-M. Pan, The order of the automorphism group of finite abelian group, J. Yunnan Univ. Nat. Sci. 26 (2004) 370-372.

[4] K. Shoda, Uber die Automorphismen einer endlischen Abelschen Gruppe, Math. Ann. 100 (1928) 674-686.

Department of Mathematics, Texas A\&M University, College Station, TX 77843

E-mail address: chillar@math.tamu.edu

Department of Mathematics, University of California, Berkeley, CA 94720

E-mail address: drhea@math.berkeley.edu 